\title{
Strong back-action of a linear circuit on a single electronic quantum channel
}

\author{
F. D. Parmentier, A. Anthore ${ }^{\star}$, S. Jezouin, H. le Sueur ${ }^{\dagger}$, U. Gennser, A. Cavanna, D. Mailly \\ and F. Pierre ${ }^{\star}$
}

The question of which laws govern electricity in mesoscopic circuits is a fundamental matter that also has direct implications for the quantum engineering of nanoelectronic devices. When a quantum-coherent conductor is inserted into a circuit, its transport properties are modified; in particular, its conductance is reduced because of the circuit back-action. This phenomenon, known as environmental Coulomb blockade, results from the granularity of charge transfers across the coherent conductor ${ }^{1}$. Although extensively studied for a tunnel junction in a linear circuit ${ }^{2-5}$, it is only fully understood for arbitrary short coherent conductors in the limit of small circuit impedances and small conductance reduction ${ }^{6-8}$. Here, we investigate experimentally the strong-back-action regime, with a conductance reduction of up to $90 \%$. This is achieved by embedding a single quantum channel of tunable transmission in an adjustable on-chip circuit of impedance comparable to the resistance quantum $R_{\mathrm{K}}=h / \mathrm{e}^{2}$ at microwave frequencies. The experiment reveals significant deviations from calculations performed in the weak back-action framework ${ }^{6,7}$, and is in agreement with recent theoretical results ${ }^{9,10}$. Based on these measurements, we propose a generalized expression for the conductance of an arbitrary quantum channel embedded in a linear circuit.

The transport properties of a coherent conductor depend on the surrounding circuit. First, electronic quantum interferences blend the conductor with its vicinity, resulting in a different coherent conductor (see for example ref. 11). Furthermore, the circuit backaction modifies the full counting statistics of charge transfers across coherent conductors ${ }^{9,10,12}$. This mechanism, which is our concern here, results in violations of the classical impedance composition laws even for distinct circuit elements, separated by more than the electronic phase coherence length. The present experimental work investigates the strong circuit back-action on the conductance of an arbitrary electronic quantum channel.

The circuit back-action originates from the granularity in the transfer of charges across a coherent conductor. As a result of Coulomb interactions, an excitation by these current pulses of the circuit electromagnetic modes is possible, which impedes the charge transfers and therefore reduces the conductance of the coherent conductor. This environmental Coulomb blockade is best understood in the limit of a tunnel junction embedded in a circuit of very high series impedance, which is of particular importance for single-electron devices ${ }^{13}$. In this limit, each time an electron tunnels across the junction, its charge stays a very long time on the capacitor $C$ inherent to the junction's geometry. Consequently, a charging energy $e^{2} / 2 C$ has to be paid. As this energy is not available at low a

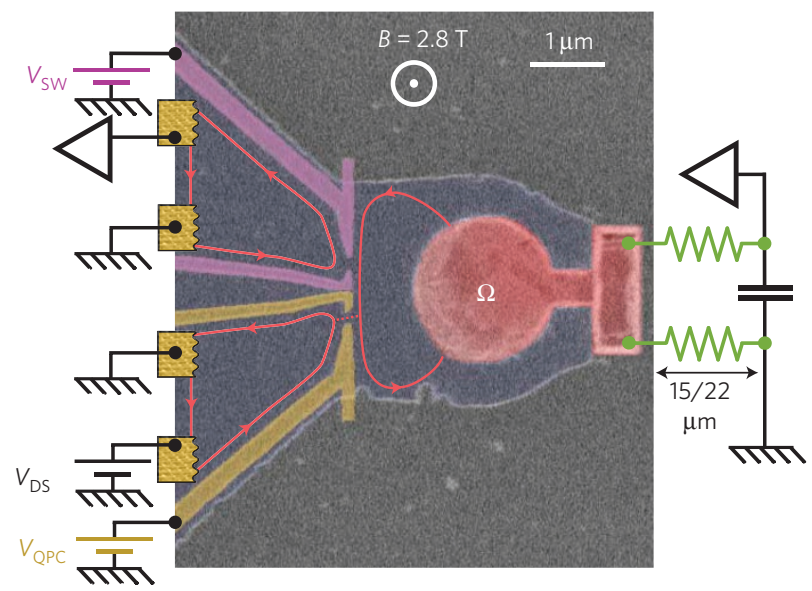

b
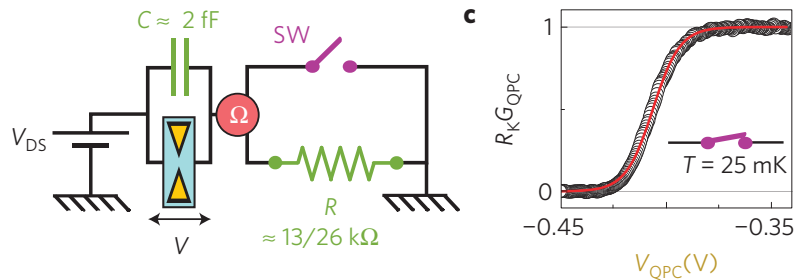

Figure 1 | Measured devices. a, SEM micrograph of the $R=26 \mathrm{k} \Omega$ sample. The bottom-left metal split gate (yellow) is used to tune the studied QPC. The outer-edge channel, shown as a red line, is partially transmitted at the QPC. A small ohmic contact labelled $\Omega$ (red) is used to connect the 2DEG (light blue) with the series chromium wires symbolized by green resistors. The top-left split gate (violet) realizes a switch to short-circuit the on-chip impedance. $\mathbf{b}$, Schematic of the equivalent circuit, with $C$ the parallel geometrical capacitance. c, Conductance $G_{Q P C}$ of the bottom left QPC in a versus the applied gate voltage $V_{Q P C}$, for a short-circuited impedance.

voltages and temperatures, the tunnelling of electrons is blocked and the tunnel junction's conductance vanishes. One speaks of 'static' Coulomb blockade, because the circuit's dynamical response can be ignored. Beyond this limit, if the circuit response time $\tau$ is short enough, the charging energy becomes ill-defined, with an uncertainty $\Delta E \approx h / \tau \gtrsim e^{2} / 2 C$. This 'dynamical' Coulomb blockade regime corresponds to quantum fluctuations of the charge on the capacitor that are comparable to the electron charge $e$. It is therefore essential to consider the circuit as a quantum object. For a resistor $R$ in series with the tunnel junction, the crossover 

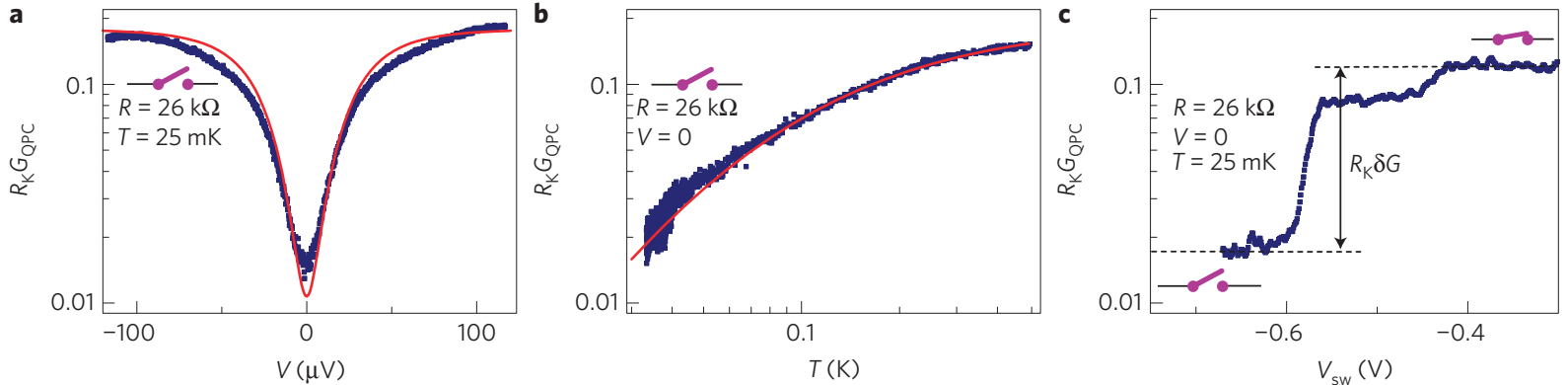

Figure 2 | Back-action signal versus prediction in the tunnel limit, for the $\boldsymbol{R}=\mathbf{2 6} \mathbf{k} \mathbf{\Omega}$ sample. Predictions (see text) are shown as continuous lines and data as symbols. a, Differential conductance $G_{Q P C}$ versus $V$ at $T=25 \mathrm{mK}$ with the switch open. $\mathbf{b}, G_{\mathrm{QPC}}$ versus temperature at $V=0$ with the switch open. c, $G_{\mathrm{QPC}}$ versus switch gate voltage $V_{\mathrm{SW}}$ at $V=0$ and $T=25 \mathrm{mK}$. The series resistance, $R=26 \mathrm{k} \Omega$ at $V_{\mathrm{SW}}<-0.6 \mathrm{~V}$, is short-circuited at $V_{\mathrm{SW}}>-0.4 \mathrm{~V}$.

between the static and the dynamical Coulomb blockade is at $R \approx R_{\mathrm{K}} \equiv h / e^{2} \simeq 25.8 \mathrm{k} \Omega$. Importantly, the conductance can also be fully suppressed in the dynamical regime, at sufficiently low energy.

The environmental Coulomb blockade was first studied on small, opaque tunnel junctions embedded in linear circuits ${ }^{2-5}$. The studies were later extended to tunnel junctions of larger conductance $^{14}$ and size ${ }^{15}$, and to the high-frequency domain ${ }^{16}$. To go beyond tunnel junctions, a major theoretical difficulty is that a general coherent conductor, with electronic channels of arbitrary transmission probabilities, cannot be handled as a small perturbation to the circuit. This difficulty was first overcome in the limit of low-impedance linear circuits with a small back-action. In this case, the striking prediction ${ }^{6,7}$ and observation ${ }^{8}$ are that the circuit back-action on the conductance is directly proportional to the amplitude of quantum shot noise in the absence of the circuit. However, the even more important and challenging regime of strong back-action remains mostly unexplored and unsolved for arbitrary coherent conductors, despite important advances in that direction $^{9,12,17-19}$ and a powerful link established with the Luttinger physics of interacting $1 \mathrm{D}$ conductors ${ }^{10}$. The present experimental work investigates this regime on a tunable quantum point contact (QPC) embedded in an on-chip circuit of impedance comparable to $R_{\mathrm{K}}$, beyond reach of perturbative theoretical treatments, resulting in relative reductions of the QPC conductance of up to $90 \%$.

The samples are constituted of three basic elements (see Fig. 1a,b): a tunable single electronic channel as a test-bed for coherent conductors, an on-chip dissipative environment and a switch to short-circuit the dissipative environment.

We emulate any single-channel short coherent conductor with a tunable QPC formed by field effect in a buried $\mathrm{GaAs} / \mathrm{Ga}(\mathrm{Al})$ As twodimensional electron gas (2DEG), using a surface metallic split gate biased at $V_{\mathrm{QPC}}$ (bottom split gate in Fig. 1a). A single-channel short coherent conductor is characterized by the 'intrinsic' quantum channel transmission probability $\tau_{\infty} \equiv R_{\mathrm{K}} G_{\infty} \in[0,1]$, with $G_{\infty}$ the coherent conductor's conductance in the absence of circuit back-action. The single step followed by a well-defined $1 / R_{\mathrm{K}}$ plateau of the QPC conductance $G_{\mathrm{QPC}}\left(V_{\mathrm{QPC}}\right)$ (symbols in Fig. 1c) shows that the studied QPC can be operated in the single-channel regime, and that its transmission probability can be varied continuously from 0 to 1 . The canonical QPC behaviour is confirmed by fitting the measured $G_{\mathrm{OPC}}\left(V_{\mathrm{OPC}}\right)$ with the standard saddle-point model of a QPC (ref. 20; continuous line in Fig. 1c). Note that it is important to break spin degeneracy to first study a single electronic channel. Otherwise, the additional channels would partly shunt the surrounding circuit ${ }^{14,21}$. For this purpose, we applied a strong perpendicular magnetic field $B=2.8 \mathrm{~T}$ corresponding to the integer quantum Hall effect at filling factor 4. Consequently, the current propagates at the edges along four copropagating edge channels. The studied outer edge channel is shown in Fig. 1a as a red line, with an arrow indicating the propagation direction.

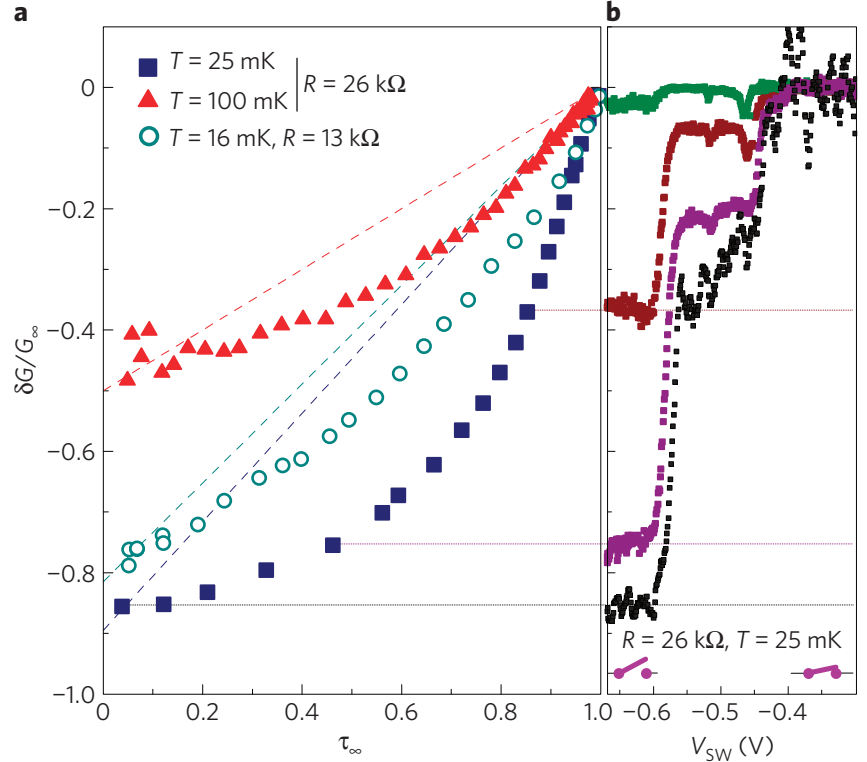

Figure 3 | Environment back-action versus transmission probability. a, Measured relative back-action amplitude $\delta G / G_{\infty}$ (symbols) versus 'intrinsic' transmission probability $\tau_{\infty}$. The data with $R=26 \mathrm{k} \Omega$ are shown for $T=25 \mathrm{mK}$ (solid square) and $T=100 \mathrm{mK}$ (solid triangle). Those with $R=13 \mathrm{k} \Omega$ are shown for $T=18 \mathrm{mK}$ (circle). The dashed lines represent the $\left(1-\tau_{\infty}\right)$ behaviour predicted in the limit of small environmental impedances. b, Sweeps $\delta G / G_{\infty}\left(V_{S W}\right)$ measured at $\tau_{\infty}=\{0.038,0.462,0.853,0.987\}$, respectively from bottom to top, in the $26 \mathrm{k} \Omega$ sample for $T=25 \mathrm{mK}$.

The three other edge channels (not shown) are always fully reflected at the QPC.

The second element is the surrounding circuit of the QPC, of large dissipative impedance $\operatorname{Re}[Z(\omega)] \sim R_{\mathrm{K}}$ up to microwave frequencies $\omega \sim k_{\mathrm{B}} T / h \sim 1 \mathrm{GHz}$. This is achieved with a nanofabricated on-chip environment modelled by the linear RC circuit in Fig. 1b. The series resistances $R$ is $26 \mathrm{k} \Omega$ for the sample shown in Fig. 1a and $13 \mathrm{k} \Omega$ for a second sample. It is realized by two parallel thin chromium wires of identical lengths $L=22 \mu \mathrm{m}(15 \mu \mathrm{m})$ for $R=26 \mathrm{k} \Omega(13 \mathrm{k} \Omega)$ deposited at the surface. These chromium wires can be described as macroscopic linear resistors (see Supplementary Information). The parallel capacitance $C$ in Fig. $1 \mathrm{~b}$ corresponds to the shunt capacitor to a.c. ground of the area delimited by the metal split gates and the series chromium wires. To avoid a capacitive short-circuit of the series resistance at the relevant microwave frequencies, this area must be minimized. For this purpose, the buried 2DEG is connected to the chromium resistors at the surface with a micrometre-scale AuGeNi ohmic contact (labelled $\Omega$ in 

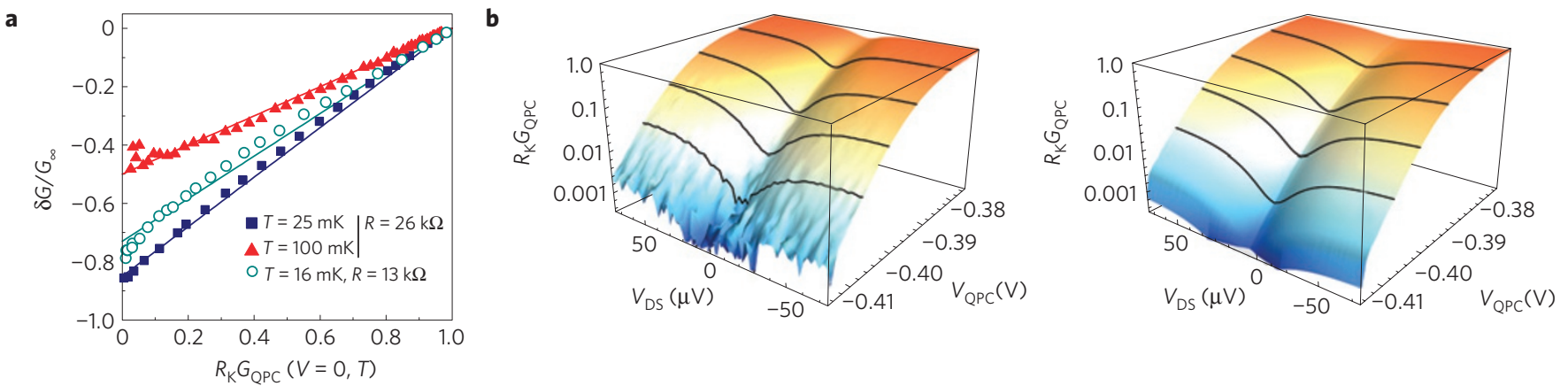

Figure 4 | Comparison between data and extended strong back-action predictions. a, $\delta G / G_{\infty}$ (same datasets as Fig. 3a) plotted as a function of the conductance $G_{Q P C}(V=0, T)$ in the presence of back-action. The straight continuous lines are guides for the eyes. b, Normalized QPC differential conductance $R_{K} G_{Q P C}$ plotted on a log scale as a function of the QPC gate voltage $V_{\mathrm{QPC}}$ and the applied drain-source voltage $V_{\mathrm{DS}}$. The continuous lines correspond to different values of the 'intrinsic' transmission probability $\tau_{\infty}$, from top to bottom $\{0.78,0.37,0.097,0.018\}$. Left panel, conductance measured with $R=26 \mathrm{k} \Omega$; right panel, calculations using equation (1) with $\tau_{\infty}\left(V_{\mathrm{QPC}}\right)$ set to the measured $R_{\mathrm{K}} G_{\mathrm{QPC}}\left(V_{\mathrm{QPC}}, V_{\mathrm{DS}}=100 \mu \mathrm{V}\right)$.

Fig. 1a). This micrometre-scale contact also plays the role of a floating electron reservoir, which breaks the quantum coherence between electrons emitted and arriving at the studied QPC.

The third element is a switch that allows us to suppress the back-action of the environment by short-circuiting it. This switch is controlled by the voltage $V_{\text {SW }}$ applied to the top split gate in Fig. 1a. A second voltage amplifier (top left in Fig. 1a) is used to monitor the switch's conductance.

In the present experiment, the reduction $\delta G$ of the QPC conductance $G_{\mathrm{QPC}}$ by the circuit back-action is extracted by three different methods: We measure $G_{\mathrm{QPC}}$ as a function of either the d.c. voltage $V$ across the QPC (Fig. 2a), the temperature (Fig. 2b), or the gate voltage $V_{\mathrm{SW}}$ controlling the switch (Fig. 2c). In the first two methods, traditionally used to investigate the Coulomb blockade, $\delta G \equiv G_{\mathrm{QPC}}(V=0, T)-G_{\infty}$ is obtained by assuming that $G_{\mathrm{QPC}}$ converges towards its 'intrinsic' conductance $G_{\infty}$ for $\mathrm{eV}$ or $k_{\mathrm{B}} T$ much larger than $h / R C$ and $e^{2} / 2 C$. In the third method, $G_{\infty}$ is obtained from the QPC conductance measured with a shortcircuited environmental impedance. This last, more direct method yields the back-action signal without any particular assumption on its energy dependence, and avoids possible sources of error related to the transmission energy dependence, sample heating, or the QPC stability over long times.

Figure 2 illustrates the three methods for the same sample of series resistance $R=26 \mathrm{k} \Omega$, and with the QPC set to similar low transmissions. In this near-tunnel limit, the measured voltage and temperature dependence of $G_{\mathrm{OPC}}$, shown as symbols in Fig. 2a and $b$, can be compared to the known predictions for tunnel junctions ${ }^{1}$. The calculations, plotted as continuous lines, were performed within the simplified RC model depicted in Fig. $1 \mathrm{~b}$. The temperature $T$ was set to that of the dilution fridge mixing chamber, $R=26 \mathrm{k} \Omega$ to the measured value of the on-chip series resistance, and the parallel geometrical capacitance $C$ to the value $C=2 \mathrm{fF}$ obtained by finite element numerical simulations. The only fit parameter is the transmission in the absence of backaction $\tau_{\infty}=0.18$ (Fig. 2a) and 0.19 (Fig. 2b). Figure 2c shows $G_{\mathrm{QPC}}(V=0, T=25 \mathrm{mK})$ versus the voltage $V_{\mathrm{SW}}$ controlling the switch to short-circuit the environment. The capacitive crosstalk between the switch gates and the QPC gates was first calibrated for each sample, then compensated for when sweeping $V_{\mathrm{SW}}$ (see Supplementary Information). For $V_{\mathrm{SW}}<-0.6 \mathrm{~V}$, the conductance across the switch is zero, and the measured $G_{\mathrm{QPC}}$ corresponds to the conductance reduced by the environmental back-action. As $V_{\mathrm{SW}}$ is increased, the switch's conductance increases in steps corresponding to the successive edge channel transmission. The environmental back-action is found to be suppressed by fully transmitting the two outer edge channels across the switch (see
Supplementary Information); the corresponding QPC conductance measured at $V_{\mathrm{SW}} \in[-0.4,-0.3] \mathrm{V}$ is taken as $G_{\infty}$. We stress that the conductance reductions $\delta G$ obtained from all three methods are consistent with one another, and that we find a good agreement between data and theoretical predictions in the tunnel limit for a known surrounding circuit. This provides strong support for our interpretation of $\delta G$ in terms of environmental back-action. We have now established the experimental principle with a tunnel QPC, and demonstrated the strong back-action regime with a conductance reduction of $90 \%$.

Next, we investigate the circuit back-action on an arbitrary single-channel coherent conductor characterized by its 'intrinsic' transmission probability $\tau_{\infty}$. Figure 3 a shows as symbols, for both samples, the measured relative reduction of the QPC conductance $\delta G / G_{\infty}$ due to the circuit back-action when the switch is open, as a function of $\tau_{\infty}$. Figure $3 \mathrm{~b}$ shows the sweeps $\delta G / G_{\infty}\left(V_{\mathrm{SW}}\right)$ at $\tau_{\infty}=\{0.038,0.462,0.853,0.987\}$ for the $R=26 \mathrm{k} \Omega$ sample at $25 \mathrm{mK}$. First, we observe that $\left|\delta G / G_{\infty}\right|$ is largest in the tunnel limit and diminishes monotonously towards zero as $\tau_{\infty}$ increases towards full transmission. However, contrary to predictions and observations in the limit of small environmental impedances ${ }^{6-8},\left|\delta G / G_{\infty}\right|$ is not proportional to $\left(1-\tau_{\infty}\right)$ in the full range $\tau_{\infty} \in[0,1]$ (dashed lines), but markedly larger at intermediate $\tau_{\infty}$. As seen by comparing the data at $T=25 \mathrm{mK}$ and $100 \mathrm{mK}$ for the $R=26 \mathrm{k} \Omega$ sample, when the temperature increases, $\left|\delta G / G_{\infty}\right|$ and the deviations to a $\left(1-\tau_{\infty}\right)$ dependence decrease.

Remarkably, we observe that the back-action correction to the conductance $\delta G / G_{\infty}$ is instead proportional to $\left(1-R_{\mathrm{K}} G_{\mathrm{QPC}}(V=0, T)\right)$, for all series resistances and temperatures. This is demonstrated, within experimental uncertainties, in Fig. 4a by plotting $\delta G / G_{\infty}$ now as a function of $R_{\mathrm{K}} G_{\mathrm{QPC}}(V=0, T)$. This proportionality can be written as $\delta G / G_{\infty}=\left(1-R_{\mathrm{K}} G_{\mathrm{QPC}}\right) E_{\mathrm{B}}$, where $E_{\mathrm{B}} \equiv \lim _{G_{\infty} \rightarrow 0} \delta G / G_{\infty}$ is the relative circuit back-action for a small tunnel junction embedded in the same circuit. Using the environmental Coulomb blockade framework for tunnel junctions ${ }^{1}$, $E_{\mathrm{B}}(Z, V, T)$ can be calculated for arbitrary circuit impedances $Z$, bias voltages $V$ and temperatures $T$. Consequently, solving the above equation for $G_{\mathrm{QPC}}$ allows us to propose a generalized expression for the conductance of a single electronic channel of arbitrary transmission embedded in a linear environment of arbitrary impedance (see also Supplementary Information):

$$
G_{\mathrm{QPC}}(V, T)=\frac{\tau_{\infty}}{R_{\mathrm{K}}} \frac{1+E_{\mathrm{B}}(Z, V, T)}{1+\tau_{\infty} E_{\mathrm{B}}(Z, V, T)}
$$

We further tested the proposed equation (1) by comparing in Fig. $4 \mathrm{~b}$ the measured (left panel) and calculated (right panel) QPC conductance versus the applied bias voltage $V_{\mathrm{DS}}$ and the gate 
voltage $V_{\mathrm{OPC}}$ for $R=26 \mathrm{k} \Omega$. The calculations were performed with equation (1) using $\tau_{\infty}\left(V_{\mathrm{QPC}}\right) \simeq R_{\mathrm{K}} G_{\mathrm{QPC}}\left(V_{\mathrm{QPC}}, V_{\mathrm{DS}}=100 \mu \mathrm{V}\right)$. We find a good agreement between data and theory for $\tau_{\infty}<0.5$, illustrating the validity of this formula even at finite bias voltage. Note that, for $\tau_{\infty} \gtrsim 0.5$, we find that the measured dip in $R_{\mathrm{K}} G_{\mathrm{QPC}}\left(V_{\mathrm{DS}}\right)$ is significantly narrower than calculations. However, this deviation can be accounted for by including the significant sample heating by the d.c. current within a simplified model based on the Wiedemann-Franz law (see Supplementary Information).

Moreover, equation (1) agrees with a recent theoretical prediction using a renormalization-group approach ${ }^{9}$ (see Supplementary Information), and generalizes it to arbitrary impedances, beyond resistances small compared with $R_{\mathrm{K}}$.

Equation (1) could be understood as a direct link between the conductance reduction by the circuit back-action and the quantum shot noise in the presence of the circuit. The bridge established for a purely resistive environment between Luttinger physics and the environmental Coulomb blockade suggests that $\delta G / G_{\infty}$ remains proportional to the amplitude of quantum shot noise for arbitrary series impedances ${ }^{10}$. As pointed out in ref. 10, the quantum shot noise is now strongly modified by the environmental back-action. Although there is no fully developed theoretical framework, the experimental observation $\delta G / G_{\infty}=\left(1-R_{\mathrm{K}} G_{\mathrm{QPC}}\right) E_{\mathrm{B}}$, from which equation (1) is derived, would correspond to a quantum shot noise spectral density of the current $S_{\mathrm{I}}$ in the presence of back-action that verifies $\mathrm{d} S_{\mathrm{I}} / \mathrm{d} V=2 e G_{\mathrm{QPC}}\left(1-R_{\mathrm{K}} G_{\mathrm{QPC}}\right)$. Significantly, the same expression is verified in the absence of circuit back-action ${ }^{22,23}$, but using the 'intrinsic' transmission probability $\tau_{\infty}$ instead of the measured transmission probability $R_{\mathrm{K}} G_{\mathrm{QPC}}$. These relations can be derived exactly in the special case $Z(\omega)=R_{\mathrm{K}}$ (I. Safi, private communication: a detailed comparison with equation (1) will be published elsewhere).

To conclude, we explored the strong back-action of a linear circuit on an arbitrary, single-channel, short coherent conductor. The results suggest the generalized expression equation (1) for the environmental back-action, which remains to be derived theoretically. This experiment opens the path for further investigations of the quantum laws of electricity in nanocircuits. These include the investigation of circuits with coherent conductors in which the environmental back-action can coexist with other phenomena such as the Kondo effect ${ }^{24}$, as well as the investigation of the circuit back-action on the full statistics of charge transfers across a coherent conductor ${ }^{9,10,12}$.

\section{Methods}

The measurements were performed in a dilution refrigerator down to $T=16 \mathrm{mK}$, on two samples tailored in a typical 2DEG. The 2DEG of density $2.5 \times 10^{15} \mathrm{~m}^{-2}$ and mobility $55 \mathrm{~m}^{2} \mathrm{~V}^{-1} \mathrm{~s}^{-1}$ is buried $94 \mathrm{~nm}$ deep in a $\mathrm{GaAs} / \mathrm{Ga}(\mathrm{Al})$ As heterojunction. The measured differential conductances were obtained by standard lock-in techniques at frequencies below $100 \mathrm{~Hz}$ with custom-made ultra-low noise electronics. To measure independently the QPC conductance and the series resistance $R$, and also to test the small ohmic contact, the end of one of the chromium wires realizing $R$ is connected at room temperature to a high-impedance voltage amplifier (see Fig. 1a). As a result of antenna effects, the impedance of the line towards the amplifier is reduced at the relevant microwave frequencies to approximately the vacuum impedance $377 \Omega \ll R_{\mathrm{K}}$. This is symbolized in Fig. 1a by a capacitor in parallel with the top right amplifier. Further details are given in the Supplementary Information.

Received 4 May 2011; accepted 12 August 2011; published online 18 September 2011

\section{References}

1. Ingold, G-L. \& Nazarov, Y. Single Charge Tunneling; Coulomb Blockade Phenomena in Nanostructures Ch. 2 (Plenum, 1992).

2. Devoret, M. H. et al. Effect of the electromagnetic environment on the Coulomb blockade in ultrasmall tunnel junctions. Phys. Rev. Lett. 64, 1824-1827 (1990).
3. Girvin, S. M., Glazman, L. I., Jonson, M., Penn, D. R. \& Stiles, M. D. Quantum fluctuations and the single-junction Coulomb blockade. Phys. Rev. Lett. 64, 3183-3186 (1990).

4. Cleland, A. N., Schmidt, J. M. \& Clarke, J. Influence of the environment on the Coulomb blockade in submicrometer normal-metal tunnel junctions. Phys. Rev. B 45, 2950-2961 (1992).

5. Holst, T., Esteve, D., Urbina, C. \& Devoret, M. H. Effect of a transmission line resonator on a small capacitance tunnel junction. Phys. Rev. Lett. 73, 3455-3458 (1994).

6. Yeyati, A. L., Martin-Rodero, A., Esteve, D. \& Urbina, C. Direct link between Coulomb blockade and shot noise in a quantum-coherent structure. Phys. Rev. Lett. 87, 046802 (2001).

7. Golubev, D. S. \& Zaikin, A. D. Coulomb interaction and quantum transport through a coherent scatterer. Phys. Rev. Lett. 86, 4887-4890 (2001).

8. Altimiras, C., Gennser, U., Cavanna, A., Mailly, D. \& Pierre, F. Experimental test of the dynamical Coulomb blockade theory for short coherent conductors. Phys. Rev. Lett. 99, 256805 (2007).

9. Kindermann, M. \& Nazarov, Y. V. Interaction effects on counting statistics and the transmission distribution. Phys. Rev. Lett. 91, 136802 (2003).

10. Safi, I. \& Saleur, H. One-channel conductor in an ohmic environment: Mapping to a Tomonaga-Luttinger liquid and full counting statistics. Phys. Rev. Lett. 93, 126602 (2004).

11. Umbach, C. P., Santhanam, P., van Haesendonck, C. \& Webb, R. A. Nonlocal electrical properties in mesoscopic devices. Appl. Phys. Lett. 50, 1289-1291 (1987).

12. Golubev, D. S., Galaktionov, A. V. \& Zaikin, A. D. Electron transport and current fluctuations in short coherent conductors. Phys. Rev. B 72, 205417 (2005).

13. Devoret, M. H., Esteve, D. \& Urbina, C. Single-electron transfer in metallic nanostructures. Nature 360, 574-553 (1992).

14. Joyez, P., Esteve, D. \& Devoret, M. H. How is the Coulomb blockade suppressed in high-conductance tunnel junctions? Phys. Rev. Lett. 80, 1956-1959 (1998).

15. Pierre, F. et al. Electrodynamic dip in the local density of states of a metallic wire. Phys. Rev. Lett. 86, 1590-1593 (2001).

16. Hofheinz, M. et al. Bright side of the Coulomb blockade. Phys. Rev. Lett. 106, 217005 (2011).

17. Matveev, K. A., Yue, D. \& Glazman, L. I. Tunneling in one-dimensional non-Luttinger electron liquid. Phys. Rev. Lett. 71, 3351-3354 (1993).

18. Molenkamp, L. W., Flensberg, K. \& Kemerink, M. Scaling of the Coulomb energy due to quantum fluctuations in the charge on a quantum dot. Phys. Rev. Lett. 75, 4282-4285 (1995).

19. Nazarov, Y. V. Coulomb blockade without tunnel junctions. Phys. Rev. Lett. 82, 1245-1248 (1999).

20. Büttiker, M. Quantized transmission of a saddle-point constriction. Phys. Rev. B 41, 7906-7909 (1990).

21. Bagrets, D. A. \& Nazarov, Y. V. Full current statistics in the regime of weak coulomb interaction. Phys. Rev. Lett. 94, 056801 (2005).

22. Martin, T. \& Landauer, R. Wave-packet approach to noise in multichannel mesoscopic systems. Phys. Rev. B 45, 1742-1755 (1992).

23. Blanter, Y. M. \& Büttiker, M. Shot noise in mesoscopic conductors. Phys. Rep. 336, 1-166 (2000).

24. Florens, S., Simon, P., Andergassen, S. \& Feinberg, D. Interplay of electromagnetic noise and Kondo effect in quantum dots. Phys. Rev. B 75,155321 (2007).

\section{Acknowledgements}

The authors gratefully acknowledge Y. Nazarov, the Quantronics group and I. Safi for discussions, F. Lafont for his contribution to the experiment, and L. Couraud and C. Ulysse for their contributions to the nano-fabrication. This work was supported by the ERC (ERC-2010-StG-20091028, \#259033), the ANR (ANR-09-BLAN-0199) and NanoSci-ERA (ANR-06-NSCI-001).

\section{Author contributions}

A.A., F.D.P., F.P. and S.J. carried out the experimental work and theoretical analysis; A.A., F.P. and H.l.S. carried out nano-fabrication and sample design with inputs from D.M.; A.C. and U.G. carried out heterojunction growth; A.A., F.D.P., F.P. and U.G. prepared the manuscript with inputs from coauthors; F.P planned and supervised the project.

\section{Additional information}

The authors declare no competing financial interests. Supplementary information accompanies this paper on www.nature.com/naturephysics. Reprints and permissions information is available online at http://www.nature.com/reprints. Correspondence and requests for materials should be addressed to A.A. or F.P. 